\title{
Hybrid composite sandwich panels under blast and impact loading
}

\author{
Emily Rolfe ${ }^{1}$, Hari Arora ${ }^{2}$, Paul A. Hooper ${ }^{1}$, and John P. Dear ${ }^{1, *}$ \\ ${ }^{1}$ Department of Mechanical Engineering, Imperial College London, London, SW7 2AZ \\ ${ }^{2}$ Zienkiewicz Centre for Computational Engineering, Swansea University, Wales, SA1 8EN
}

\begin{abstract}
Naval vessels may undergo high strain rate loading, including impact, wave slamming and blast loading. Predicting the behaviour of composite sandwich structures to such loading is complicated, hence representative experiments are required. Two panels with hybrid carbon- and glass-fibre skins were fabricated and subjected to full-scale air blast loading. The panels were $1.7 \times 1.5 \mathrm{~m}$ in size and were subjected to a 100 $\mathrm{kg}$ nitromethane charge at a stand-off distance of $15 \mathrm{~m}$. 3D Digital Image Correlation (DIC) was implemented behind each of the panels to record the full-field out-of-plane displacement of the panels. In addition, the panels were instrumented with foil strain gauges on the front skins to record the response of the panel side in contact with the blast. The results revealed that the combination of glass- and carbon-fibre improves the blast resilience when compared to previous blast testing on panels with exclusively glass-fibre or carbon-fibre skins. However, the order in which the glass- and carbon-fibre layers were arranged did not have a significant effect on the overall panel performance. In addition, panels with the same hybrid skins were subjected to high velocity impact testing. An aluminium projectile with $25 \mathrm{~mm}$ diameter was fired at small scale panels $(160 \mathrm{x}$ $160 \mathrm{~mm}$ ) using a laboratory gas gun at a velocity of $78 \mathrm{~ms}^{-1}$. 3D DIC was again used to record the out-of-plane displacement of these panels. In contrast to the blast experiment, the impact results showed that the order in which glass- and carbon-fibres were arranged did affect both the out-of-plane displacement and damage to the panels. The least damage occurred when glass-fibre layers were placed on the outermost layers impacted by the projectile.
\end{abstract}

\section{Introduction}

The advantageous properties possessed by composite sandwich panels ensures that they are of increasing interest in many applications including marine, automotive and wind turbine industries. In these applications, the composite sandwich panels must be designed to withstand blast, impact and high strain rate loading. A number of authors have carried out research in this field. Arora et al have subjected large-scale composite sandwich panels to a 100 $\mathrm{kg}$ nitromethane charge from a stand-off distance of 14 $\mathrm{m}$ [1]. The experiments were carried out to compare the blast resilience of glass-fibre reinforced polymer (GFRP) and carbon-fibre reinforced polymer (CFRP) skins with an identical structural foam core. The results demonstrated that both panels suffered from skin/core damage but the panel with CFRP skins exhibited a lower deflection. However, close-field underwater blast testing has shown that composite sandwich panels with CFRP skins undergo sudden brittle failure against this type of load [2]. This indicates that CFRP skins are not suitable for the range of dynamic loads that a composite sandwich panel may have to withstand. Hybridisation of composite fibres, therefore, could be advantageous under dynamic loading.

Kelly et al performed large scale blast testing on panels with GFRP skins and polypropylene (PP) interlayers [3]. The results revealed that the addition of the PP interlayers

\footnotetext{
*e-mail: j.dear@imperial.ac.uk
}

prevented front skin cracking. Further investigations into hybridising panel skins with polymer interlayers has been carried out by Gardner et al [4]. The authors subjected sandwich panels with glass-fibre skins and polyurea (PU) interlayers to shock loads of approximately 1 MPa peak incident pressure. The results demonstrated that placing the PU layer between the core and rear skin reduced deflection and improved panel integrity.

Enfedaque et al and Sevkat et al have investigated the low velocity impact performance of hybrid composite laminates $[5,6]$. Both authors concluded that impact resistance of glass-/carbon-fibre hybrid laminates was increased when glass-fibre fabrics were used in the outermost layers. Many authors support this conclusion; if fibres with highest energy absorption are placed in the outermost layers, the hybrid laminate is able to absorb more energy overall [7]. Sevkat et al also show the interaction between the dissimilar fibres leads to greater damage but a greater energy absorption capability [8]. Pandya et al showed that for a constant laminate thickness, the ballistic limit of a hybrid glass-/carbon-fibre laminate was increased by adding glass-fibre layers [9]. Randjbaran et al investigated the performance of glass-/aramid-/carbonfibre hybrids against a gas gun projectile [10]. The authors concluded that a glass-fibre layer at the front of the laminate improves impact performance.

Based on these findings composite sandwich panels with hybrid glass-/carbon-fibre skins were fabricated 


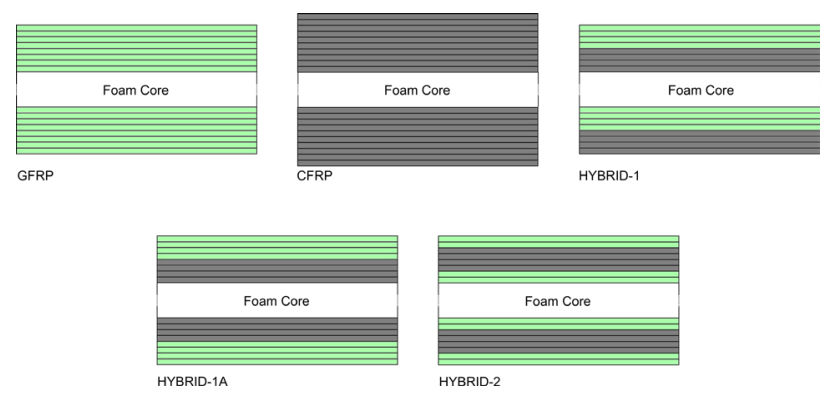

Fig. 1. Schematic of the panels subjected to high velocity impact testing.

adopting layups that were deemed to be advantageous. In order to further understand the performance of such sandwich panels, the panels were tested under representative loading. The research presented here focussed on high velocity impact testing and full scale air blast testing of the composite sandwich panels.

\section{Materials}

Five composite sandwich panels were compared under high velocity impact testing. Three panels adopted hybrid skins with differing layup orders of non-crimp fabric (NCF) glass- and carbon-fibre plies. They were tested against two benchmark panels, one with carbon-fibre skins and one with glass-fibre skins. All panels had a $20 \mathrm{~mm}$ polyehtylene terephthalate (PET) foam core. The panels were fabricated using resin infusion and were $160 \mathrm{x}$ $160 \mathrm{~mm}$ in size. The panel layups are shown in figure 1. The asymmetrical layup of Hybrid-1 aimed to take advantage of the compressive and tensile properties of glassand carbon-fibre respectively. This may only be beneficial, however, if the direction of loading is known. A symmetrical version, Hybrid-1A, was therefore fabricated. Hybrid2 aimed to contain the brittle carbon-fibre layers within the glass-fibre layers and hence maintain the integrity of the skin once the carbon-fibre has fractured. The CFRP panel was fabricated with more ply layers per skin to ensure the panel had a comparable areal density.

Following impact testing, large scale versions of Hybrid-1 and Hybrid-2 were subjected to air blast loading. For this experiment, both panels had a $25 \mathrm{~mm}$ polyvinyl chloride (PVC) foam core with density $100 \mathrm{kgm}^{-3}$. The panels were manufactured using resin infusion, they were ambient cured and held under a vacuum for 24 hours before being demolded. The panels were 1.7 x $1.5 \mathrm{~m}$ in size.

\section{Experimental Method}

The high velocity impact experiments were performed using a gas gun apparatus which accelerated a hemispherical aluminium projectile up to a velocity of $78 \mathrm{~ms}^{-1}$. The velocity of the projectile was recorded by two infrared sensors located towards the end of the gas gun barrel exit. The panel was contained within a polycarbonate and aluminium safety box and two high-speed cameras were located behind the panel outside of the safety box. These
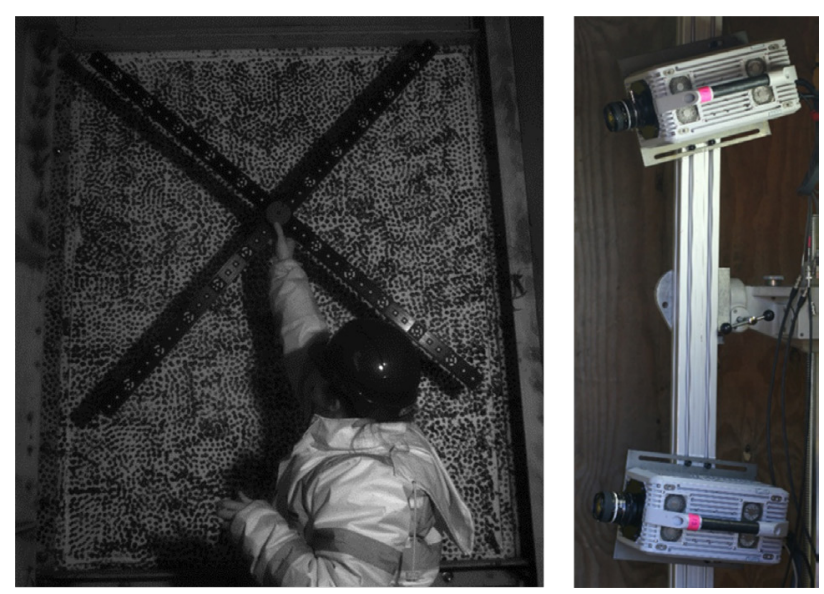

Fig. 2. Images showing the speckle pattern during calibration and setup of two high speed cameras.

cameras recorded the centre $70 \times 70 \mathrm{~mm}$ section of the panels at 40000 frames per second (fps). To facilitate DIC, the panels were spray painted matt white and a random black speckle pattern was applied to the back surface of the panel.

During the blast experiment, the panels were bolted side-by-side into a steel cubicle. $5 \mathrm{~mm}$ thick steel frames were adhered to the front and back of the panels using Sikaflex 291i marine sealing adhesive. The panels were secured to the steel cubicles using $20 \mathrm{x}$ M11 bolts around the perimeter. To prevent the sandwich panels from being crushed upon tightening of the bolts, steel tubes were placed inside the holes in the sandwich panel and sat flush against the front and rear skins of the panel. The cubicle was positioned $15 \mathrm{~m}$ away from the spherical charge which was raised to the mid-height of the panels. A pressure gauge block was also positioned $15 \mathrm{~m}$ away from the charge to record the reflected pressure experienced by the panels. A pair of high-speed cameras was arranged behind each panel within the steel cubicles to record the full-field response of the panels. Once again, to enable processing of the high-speed images as 3D DIC data, the panels were speckled. The edges of the steel cubicle were also speckled to track any movement of the boundaries. Figure 2 shows the camera setup and speckle pattern during calibration. In addition, the two panels were each instrumented with 14 foil strain gauges on the front skin. Single axis gauges were positioned at the centre of the panel and at certain locations along the edges.

\section{Results}

This section presents the results of the high velocity impact experiment followed by those of the air blast experiment. Figure 3 shows the central out-of-plane displacement for all the panels under impact at $78 \mathrm{~ms}^{-1}$. Under impact at this velocity, the CFRP panel demonstrated the lowest deflection and GFRP demonstrated the greatest deflection. As expected, the deflection of the hybrids lay between the GFRP and CFRP panels. Hybrid-1 and 


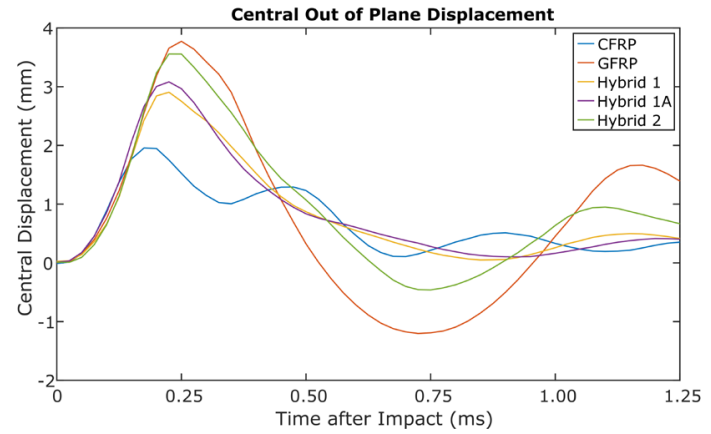

Fig. 3. Central point deflection against time under $78 \mathrm{~ms}^{-1} \mathrm{im}-$ pact.

Table 1. Details of damage to sandwich panels after $78 \mathrm{~ms}^{-1}$ impact.

\begin{tabular}{ll}
\hline Damage Type & Panel Exhibiting Damage \\
\hline Core Crushing & $\begin{array}{l}\text { CFRP/Hybrid-1/ } \\
\text { Hybrid-1A }\end{array}$ \\
Front Skin Broken Fibres & $\begin{array}{l}\text { CFRP/Hybrid-1 } \\
\text { /Hybrid-1A }\end{array}$ \\
Front Skin Delamination & $\begin{array}{l}\text { CFRP/Hybrid-1/ } \\
\text { Hybrid-1A/Hybrid-2 }\end{array}$ \\
Front Skin/Core Debonding & CFRP/GFRP/Hybrid-1/ \\
& Hybrid-1A/Hybrid-2 \\
\hline
\end{tabular}

Hybrid-1A demonstrated similar performance, the panels did not return to zero displacement before oscillating again. Hybrid-2 demonstrated higher displacement than the other two hybrid panels and Hybrid-2 exhibited a negative rebound. At this impact velocity, all panels deflected the projectile off the front skin. However, the panels did suffer from significantly different levels of damage.

Post-impact damage to the panels was analysed and shows the GFRP panel has significantly less damage than any other panels tested. The CFRP panel had a low displacement but suffered from the greatest damage. As expected, the response and damage to the hybrid panels lies between the CFRP and GFRP panels. Table 1 details the extent of the damage to each panel. Based on these results, Hybrid-1 and Hybrid-2 were selected for the full-scale air blast experiments also detailed in this paper.

The response of the panels to blast loading will now be reported. The deflection of the Hybrid-1 panel along the horizontal centre section against intervals of time are shown in figure 4(a), along with the rebound phase in 4(b). The same data is shown for the Hybrid-2 panel in figure 4(c) and (d). During the initial deflection, the panels deform in the expected bath-tub shape and the centre point of Hybrid-1 reaches a maximum displacement of $75 \mathrm{~mm}$. Sharp changes in gradient of the panel observed during the rebound phase indicate damage within the foam core and potentially within the skins. In these plots, the movement of the cubicle has been removed as it was not perfectly rigid. The maximum displacement of Hybrid-2 was $73 \mathrm{~mm}$. Despite having different skin configurations, the panels demonstrated similar overall behaviour, including comparable strain and out-of-plane dis- placement. The velocity of the central point of both panels reach a similar maximum of approximately $37 \mathrm{~ms}^{-1}$. As detailed previously, the panels were instrumented with strain gauges on the front skins to further understand the panel response. The gauges revealed that the front skins experience far greater strain than the rear skins. In addition, the strain readings show that the panel is not equally supported around the perimeter due to the cubicle design so this motion of the cubicle was removed from the panel displacement.

Table 2 shows results from the experiment described in this research along with key results from a previous blast experiment carried out by the research group [1]. The previous experiment subjected two panels to a $100 \mathrm{kgm}^{-3}$ charge at a $14 \mathrm{~m}$ stand-off distance. The panels had either GFRP or CFRP skins and a $25 \mathrm{~mm}, 100 \mathrm{kgm}^{-3}$ styrene acrylonitrile (SAN) foam core. The deflection and strain have been normalised by incident pressure or core thickness. For both the hybrids, these values are lower than for either the GFRP or CFRP panels. This indicates the combination of glass- and carbon-fibre plies in the skins reduces deflection and achieves comparable levels of strain.

\section{Discussion}

Results from the high velocity impact experiment demonstrate that layup order has an effect on the panel displacement, strain and damage under localised impact. The CFRP panels suffered from the most damage, despite the panels having a greater areal density than any other panel tested. As expected, the damage to the hybrid panels lay between the GFRP and CFRP panels. Due to the dissimilar layers in the hybrd skins, they are more likely to undergo delamination which results in more energy dissipation at the expense of greater damage. The results show there is little difference between the performance of a symmetrical and an asymmetrical panel, Hybrid-1 versus Hybrid-1A, under the loading performed in this experiment. Hybrid-2 suffers from less damage than the other hybrids, this suggests that placing carbon-fibre layers closer to the impact surface, but not as the impact surface, results in greater stiffness and resilience. This indicates that a more intricate hybridisation of the skins could further reduce delamination and damage to sandwich panels under localised impact.

The comparative air blast study revealed that the layup order does not have a significant effect on the blast response of large scale panels. The presence of both types of fibre, however, does have an effect of the panel performance. When the performance of the hybrid panels is normalised and compared to previous blast testing on panels with GFRP or CFRP skins, the hybrid panels are shown to outperform the traditional panels. Once again, this may be due to the dissimilar layers interacting and sustaining more damage. The affect of material layup in the transition from localised impact to far-field blast is of interest to the authors. 
a)

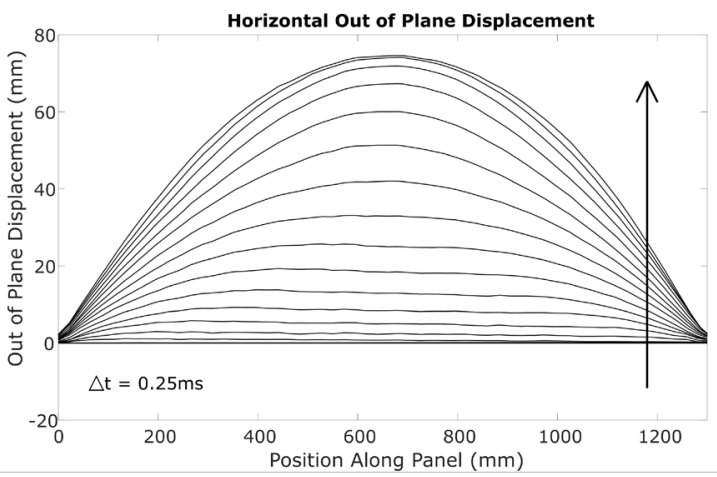

c)

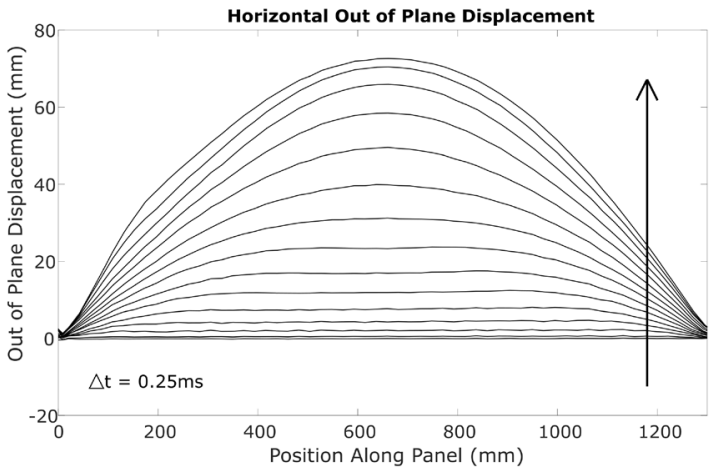

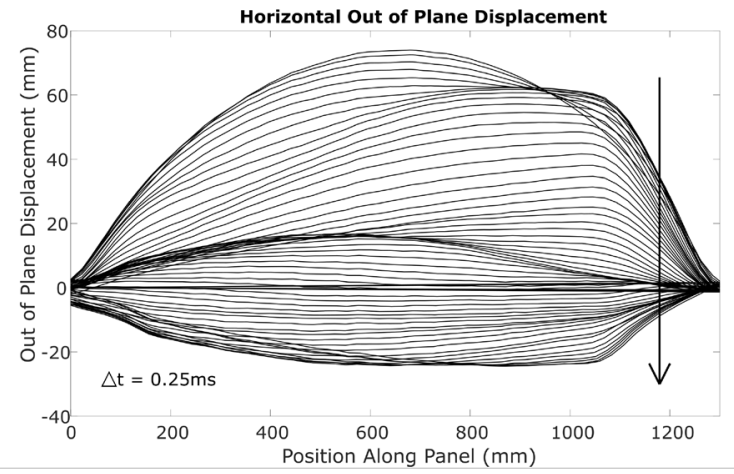

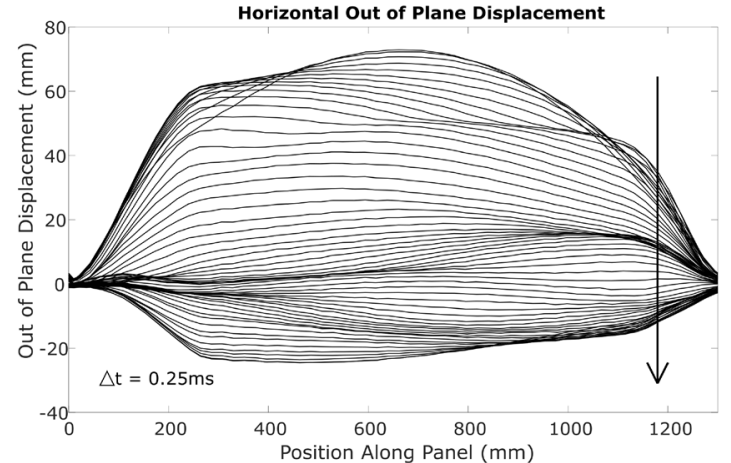

Fig. 4. Deflection at time intervals for the horizonal centre section of (a),(b) Hybrid-1 and (c),(d) Hybrid-2.

Table 2. Details of blast experiment results versus results from previous experiment.

\begin{tabular}{lllll}
\hline Panel & Description & $\mathrm{U}_{\mathrm{z} \max } / \mathrm{t}_{\text {core }}$ & $\mathrm{U}_{\mathrm{z} \max } / \mathrm{P}_{\max }$ & $\epsilon_{\mathrm{z} \max } / \mathrm{P}_{\max }$ \\
\hline Hybrid-1 & $\begin{array}{l}2 \mathrm{~mm} \text { Hybrid skins } \\
30 \mathrm{~mm} \text { PVC core }\end{array}$ & 2.50 & 0.34 & 0.003 \\
Hybrid-2 & $\begin{array}{l}2 \mathrm{~mm} \text { Hybrid skins } \\
30 \mathrm{~mm} \text { PVC core }\end{array}$ & 2.43 & 0.33 & 0.003 \\
$\mathrm{G} 25 \mathrm{~b}$ & $\begin{array}{l}2 \mathrm{~mm} \text { GFRP skins } \\
25 \mathrm{~mm} \text { SAN core }\end{array}$ & 5.60 & 0.56 & 0.006 \\
$\mathrm{C} 25 \mathrm{~b}$ & $\begin{array}{l}2 \mathrm{~mm} \text { CFRP skins } \\
25 \mathrm{~mm} \text { SAN core }\end{array}$ & 4.28 & 0.43 & 0.003 \\
& & & \\
\hline
\end{tabular}

\section{Conclusions}

The following bullet points summarise the main findings from this research:

- Layup order of glass-/carbon-fibre plies affects panel behaviour during localised impact loading,

- Placing carbon-fibre layers closer to the impact skin, however not as the impact skin itself, results in greater panel stiffness and less skin delamination,

- The dissimilar layers in the hybrid skins make them more prone to delamination which results in more damage but also greater energy absorption,

- Under blast loading the combination of glass- and carbon-fibre layers decreases deflection compared to GFRP or CFRP skins when normalised by blast pressure or panel thickness.

Much appreciated is the strong support received from Dr Yapa Rajapakse from the Office of Naval Research for sponsoring Emily Rolfe, Dr Mark Kelly and Dr Hari Arora during their
PhDs. The authors would also like to thank EPSRC for supporting Emily Rolfe during her PhD.

\section{References}

1. H. Arora, P. Hooper, L.P. Del, H. Yang, S. Chen, J. Dear, The International Journal of Multiphysics 6, 199 (2012)

2. E. Rolfe, M. Kelly, H. Arora, P.A. Hooper, J.P. Dear, Composites Part B 129, 26 (2017)

3. M. Kelly, H. Arora, a. Worley, M. Kaye, P.D. Linz, P.a. Hooper, J.P. Dear, Experimental Mechanics pp. 1-22 (2015)

4. N. Gardner, E. Wang, P. Kumar, A. Shukla, Experimental Mechanics 52, 119 (2012)

5. E. Sevkat, B. Liaw, F. Delale, B.B. Raju, Composites Part A: Applied Science and Manufacturing 40, 1090 (2009) 
6. A. Enfedaque, J.M. Molina-Aldareguía, F. Gálvez, C. González, J. LLorca, Journal of Composite Materials 44, 3051 (2010)

7. Y. Swolfs, L. Gorbatikh, I. Verpoest, Composites Part A: Applied Science and Manufacturing 67, 181 (2014)
8. E. Sevkat, B. Liaw, F. Delale, B.B. Raju, Composites Part B: Engineering 41, 403 (2010)

9. K.S. Pandya, J.R. Pothnis, G. Ravikumar, N.K. Naik, Materials and Design 44, 128 (2013)

10. E. Randjbaran, R. Zahari, N.A. Abdul Jalil, D.L. Abang Abdul Majid, The Scientific World Journal 2014, 413753 (2014) 
